\title{
A MAGNETOELASTIC MODEL FOR VILLARI-EFFECT MAGNETOSTRICTIVE SENSORS
}

\author{
Marcelo J. Dapino* \\ Department of Mechanical Engineering \\ The Ohio State University \\ 206 West 18th Avenue \\ 2091 Robinson Lab \\ Columbus, OH 43210 \\ E-mail: dapino.1@osu.edu \\ Ph.: (614) 688-3689 \\ Fax: (614) 292-3163 \\ Ralph C. Smith \\ Department of Mathematics \\ Center for Research in Scientific Computation \\ North Carolina State University \\ Raleigh, NC 27695 \\ Frederick T. Calkins \\ Boeing Phantom Works \\ Seattle, WA 98136 \\ Alison B. Flatau \\ Department of Aerospace Engineering and Engineering Mechanics \\ Iowa State University \\ Ames, IA 50011
}

* Author to whom correspondence should be sent

Key words: magnetostriction, magnetomechanical effect, Villari effect, magnetostrictive sensor, Terfenol-D

\begin{abstract}
A magnetomechanical model for the design and control of Villari-effect magnetostrictive sensors is presented. The model quantifies the magnetization changes that a magnetostrictive material undergoes when subjected to a dc excitation field and variable stresses. The magnetic behavior is characterized by considering the Jiles-Atherton mean field theory for ferromagnetic hysteresis, which is constructed from a thermodynamic balance between the energy available for magnetic moment rotation and the energy lost as domain walls attach to and detach from pinning sites. The effect of stress on magnetization is quantified through a law of approach to the anhysteretic magnetization. Elastic properties of the sensor are incorporated by means of a wave equation that quantifies the strains and stresses arising in response to moment rotations. This yields a nonlinear PDE system for the strains, stresses and magnetization state of a magnetostrictive transducer as it drives or is driven by external loads. Because the model addresses the magnetoelastic coupling, it is applicable to both magnetostrictive sensors and actuators. Properties of the model and approximation method are illustrated by comparison with experimental data collected from a Terfenol-D sensor.
\end{abstract}




\section{INTRODUCTION}

As sensors become integrated in a growing number of industrial, defense and medical applications, the demand for improved sensor technologies for detection of various physical parameters is rapidly increasing [22]. Existing and potential applications for magnetostrictive sensors are numerous because the newer magnetostrictive materials, fabricated both in crystalline and amorphous form, can exhibit comparatively large coupling coefficients in the conversion of energy between the magnetic and elastic states. Recognizing that the magnetostrictive response can be described mathematically through invertible tensor relations, the conversion between elastic and magnetic energy is: (i) reciprocal and (ii) of linear or torsional nature depending on whether the magnetic field is longitudinal ( $x_{i i}$ directions) or circumferential ( $x_{i j}$ directions) $[12,13,25,26]$. Linear and torsional sensing mechanisms are thus possible with magnetostrictive materials. Direct and inverse magnetostrictive effects applicable to actuator and sensor modes of operation are summarized in Table 1, while constructive details on such designs can be found in [26].

Although magnetostrictive transducers provide adequate performance at low signal regimes, the demand for high performance transducers often dictates that they be driven at or near full displacement, where the presence of nonlinearities and path dependences severely compromises the accuracy of conventional linear formulations. Since the fundamental material properties needed to design and control dynamic smart material structures vary substantially as various combinations of major, minor, symmetric and asymmetric magnetization curves are traversed, no nominal design parameters of the kind defined in linear models can be determined without introducing significant simulation or characterization errors. For this fundamental reason, conventional linear models typically require the construction of laborious experimental look-up tables for properties such as elastic modulus, damping, and stiffness. But since these properties are magnetoelastic averages dependent on magnetic field, stress, temperature, and frequency, pure experimental methods present additional challenges concerning the order and time rate in which the different inputs are applied to the sensor $[1,24]$. For magnetostrictive sensors to be competitive with established sensor tech-

nologies, for instance those based on piezoelectricity, maximum performance characteristics of the kind associated with nonlinear regimes must be sought.

Table 1: Magnetostrictive effects and their inverse.

\begin{tabular}{|l|l|}
\hline Direct Effects & Inverse Effects \\
\hline \hline $\begin{array}{l}\text { Joule magnetostriction } \\
\text { Change in sample dimensions in the direction } \\
\text { of the applied field }\end{array}$ & $\begin{array}{l}\text { Villari effect } \\
\text { Change in magnetization due to applied stress }\end{array}$ \\
\hline $\begin{array}{l}\Delta E \text { effect } \\
\text { Magnetoelastic contribution to magnetocrys- } \\
\text { talline anisotropy }\end{array}$ & Magnetically induced changes in the elasticity \\
\hline $\begin{array}{l}\text { Wiedemann effect } \\
\text { Torque induced by helical anisotropy }\end{array}$ & $\begin{array}{l}\text { Matteuci effect } \\
\text { Helical anisotropy and emf induced by a } \\
\text { torque }\end{array}$ \\
\hline $\begin{array}{l}\text { Magnetovolume effect } \\
\text { Volume change due to magnetization (most } \\
\text { evident near the Curie temperature) }\end{array}$ & $\begin{array}{l}\text { Nagaoka-Honda effect } \\
\text { Change in the magnetic state due to a change } \\
\text { in the volume }\end{array}$ \\
\hline
\end{tabular}


For operation in actuators and sensors, magnetostrictive materials are customarily operated under mechanical and magnetic bias conditions. Advantages of biased operation include the ability to center operation over regions of maximum strain per unit magnetic field input, thus enabling bidirectional operation, as well as to enhance the magnetoelastic state by inducing magnetic moment alignment along magnetically easy axis perpendicular to the drive axis [9]. In addition, a mechanical bias is necessary for reliability reasons to ensure that the magnetostrictive material always operates in compression.

This paper addresses the modeling of Villari-effect sensors whose active element is a magnetostrictive element operated under mechanical and magnetic bias conditions. The proposed model is sufficiently general to provide characterization of actuation and sensing effects. In actuation mode, strains and ensuing forces arise as a result of magnetization changes which are brought about by the application of magnetic fields. Conversely, elastic deformations lead to measurable changes in magnetization thus providing a mechanism for sensing. Both modes of operation are inherently coupled through the magnetomechanical coupling and hence methods for addressing this form of coupling must be considered in models to be used in high performance transducers. Only by addressing magnetic hysteresis and magnetoelastic coupling effects in a manner consistent with the physical properties of magnetostrictive materials will models for intelligent structures based on these materials be sufficiently accurate to characterize the performance space of existing and expected applications. It is emphasized that transducer models able to characterize the magnetomechanical coupling lend themselves to the design of collocated actuators and sensors. Demonstration of the use of magnetostrictive materials for sensoriactuation applications can be found in [18, 21].

The model is formulated in the context of the transducer design shown in Figure 1, which illustrates primary components needed to fully utilize the advantageous properties of magnetostrictive materials. These components include a magnetostrictive rod, an excitation/sensing solenoid that provides the bias magnetization and sensing voltage, a prestress mechanism consisting of a bolt and a spring, a permanent magnet which is used in conjunction with the solenoid to finely adjust the bias magnetization, and magnetic couplers.

The model is presented in three steps. In the first, we consider the magnetization of a magnetostrictive rod under a fixed dc magnetizing field $H_{0}$ and a variable stress field $\sigma$. The magnetization changes arising from the application of $H_{0}$ are quantified by considering a mean field model of ferromagnetic hysteresis as originally proposed in [15]. The stress-induced component of magnetization is modeled by a law of approach to the anhysteretic magnetization as developed in [16] and extended to magnetostrictive transducers in [6]. The two components considered together provide a magnetization model based on the energy dissipated when domain walls attach to and detach from inclusions in a magnetostrictive material.

The second step involves the quantification of the magnetostriction $\lambda$ produced when the magnetostrictive rod is magnetized. The relationship between magnetization and strain is modeled by an even-terms series expansion dependent on the magnetization produced in response to a magnetic field as described in the previous step. Although $\lambda$ includes components of strain arising from the rotation of magnetic moments, that is those which give rise to magnetostrictions or active strains, it does not account for passive or material responses of the kind found in ordinary elastic materials and modeled by $s^{H} \sigma$ in the generalized Hooke's law $\varepsilon=s^{H} \sigma+d_{33} H$.

The passive effects are modeled in the third step through consideration of force balancing in the magnetostrictive rod in the form of a PDE that includes the intrinsic magnetostriction, system 
compliance, internal damping, and boundary conditions dictated by the constructive details of the transducer. The solution to this PDE provides the rod displacements and corresponding total magnetoelastic strain $\varepsilon$.

Properties of the model and approximation method are illustrated in the final section of the paper by comparison of model results with experimental data collected from a Terfenol-D sensor.

\section{MAGNETIZATION MODEL}

Components of a previous magnetomechanical model [6] are summarized here to characterize the magnetization of magnetostrictive rods as employed in sensors comprising excitation and sensing solenoids, a magnetic path, and means for mechanical preloading. Because magnetization and stress are coupled, magnetization models must be constructed by considering both entities simultaneously. Unlike the previous model, the present formulation includes the effect of externally applied forces on the elastic dynamics of the transducer as well as on the magnetic regime. Inclusion of this effect is critical to the implementation of force sensors, while also allowing the magnetization model to perform more accurately in cases where a magnetic field sensor is operated under stress created by a surrounding structure. In light of these considerations, it is assumed that the magnetization $M$ is due to a dc magnetic field $H_{0}$ in combination with a magnetomechanical component $H_{\sigma}$ associated with the stress on the magnetostrictive material $\sigma$ produced as the device drives or is driven by external loads. In order to provide a general model of the performance of magnetostrictive sensors, the magnetization is assumed to be a function of magnetic field and stress,

$$
\frac{d M}{d t}=\left(\frac{\partial M}{\partial H}\right) \frac{d H}{d t}+\left(\frac{\partial M}{\partial \sigma}\right) \frac{d \sigma}{d t} .
$$

The Jiles-Atherton domain wall model [15] is considered here as a basis for characterizing the changes in magnetization that a magnetostrictive material undergoes when subjected to a uniaxial field, given by $\partial M / \partial H$, whereas a law of approach to the anhysteretic magnetization is employed to quantify the magnetomechanical effect $\partial M / \partial \sigma[16]$. The field derivative $d H / d t$ is determined

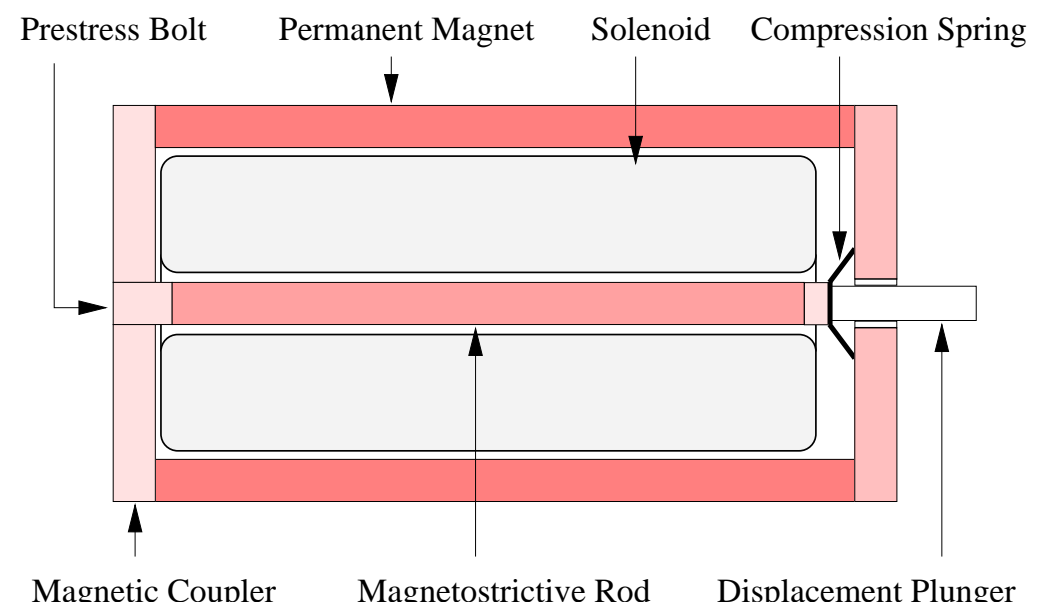

Figure 1: Magnetostrictive sensor used for model development and experimental verification. 
by the rate of application of the dc magnetic field $H_{0}$, while the stress derivative $d \sigma / d t$ must be determined from force balancing in a magnetostrictive rod as discussed later. It is noted that since $d H / d t$ is null during sensor operation, so is $d M / d t$ and the magnetization thus remains fixed at $M_{0}$. Hence, the nominal magnetization $M_{0}$ can be provided by either a solenoid or a permanent magnet as shown in Figure 1, and its value can be determined theoretically by means of the Jiles-Atherton model, or experimentally. The former approach is employed here for the sake of generality. Point $\left(H_{0}, M_{0}\right)$ on the magnetization plane represents the zero-load condition of the sensor.

\section{Constant-Stress Magnetization}

To model the differential susceptibility $\partial M / \partial H$, it is necessary to quantify the energy lost when a material in the ferromagnetic state is exposed to a cyclic uniaxial magnetic field. Under the action of a magnetic field, magnetic moments rotate toward the magnetic field direction, giving rise to the processes of domain wall motion and domain magnetization rotation [17]. Magnetic domains reorient so as to minimize the total energy, thus producing changes in the bulk magnetization.

In the idealized case of defect-free materials, on reversal of the field the magnetic moments return to their original orientations and the magnetization returns to its original value. In ferromagnetic materials, however, defects such as crystal imperfections, cracks and voids provide pinning sites to which domain walls attach since the total energy decreases as pinning sites are intersected by domain walls. For low magnetic field intensities about an equilibrium value, the domain walls remain pinned and bow in a reversible fashion, producing reversible magnetizations. But when the field intensity is sufficiently high so that the magnetic energy overcomes the pinning energy, domain walls detach irreversibly from the pinning sites and attach to remote sites. This mechanism produces energy losses which lead to magnetization hysteresis.

Assuming that no other loss mechanisms are present, the energy supplied to a ferromagnetic material is either converted into magnetostatic energy (total magnetization) or dissipated in the form of irreversible magnetization changes (hysteresis loss due to domain wall pinning). This is formulated through an energy balance in which the total magnetization is calculated from the difference between the maximum possible magnetization energy, attained in the anhysteretic state, and the energy lost to pinning. The anhysteretic magnetization is calculated using a modified formulation of the Langevin equation [15], while the energy lost to pinning is calculated in terms of a pinning coefficient $k$ that quantifies the density and strength of pinning sites. It is noted that if there is no dissipation, the magnetization must necessarily follow the minimum energy anhysteretic curve.

We first consider the anhysteretic magnetization $M_{a n}$, which is quantified using the Langevin formula $\mathcal{L}(z) \equiv \operatorname{coth}(z)-1 / z,-1<\mathcal{L}(z)<1$. As detailed in [15], $M_{a n}$ has the form

$$
M_{a n}=M_{s} \mathcal{L}\left(H_{e} / a\right),
$$

in which $M_{s}$ is the saturation magnetization and the constant $a$, representing the effective domain density, is treated as a parameter to be estimated through a least squares fit to data or through adaptive parameter identification techniques. The effective magnetic field $H_{e}$ is found by minimization of a suitable thermodynamic potential, and has the form

$$
H_{e}=H+\alpha M+H_{\sigma},
$$

where $H$ is the applied magnetic field, $\alpha M$ is the Weiss interaction field responsible for the align-

ment of neighboring magnetic moments within domains, and $H_{\sigma} \equiv 1 / \mu_{0}\left[\partial\left(\frac{3}{2} \sigma \varepsilon\right) / \partial M\right]$ is the field 
due to magnetoelastic interactions.

The differential equations for the irreversible $M_{i r r}$ and reversible $M_{r e v}$ components of magnetization $\left(M=M_{r e v}+M_{i r r}\right)$ in the material can be shown to be [15]

$$
\begin{gathered}
M_{i r r}=M_{a n}-k \delta \frac{d M_{i r r}}{d H_{e}} \\
M_{r e v}=c\left(M_{a n}-M_{i r r}\right),
\end{gathered}
$$

where the parameter $\delta$ is +1 when $d H / d t>0$ and -1 when $d H / d t<0$ to ensure that pinning losses always oppose the magnetization and $c$ is a parameter that quantifies the amount by which domain walls bulge before breaking away from pinning sites.

The total magnetization is then dictated by the superposition of the irreversible and reversible contributions given by (3) and (4) respectively,

$$
M=M_{i r r}+M_{r e v}=M_{a n}-k \delta(1-c) \frac{d M_{i r r}}{d H_{e}} .
$$

This equation leads to the total differential susceptibility $\partial M / \partial H$ upon differentiation and subsequent application of the chain rule. As detailed in [7], the total differential susceptibility has the form

$$
\frac{\partial M}{\partial H}=(1-c) \frac{M_{a n}-M_{i r r}}{\delta k-\widetilde{\alpha}\left(M_{i r r}, \sigma\right)\left(M_{a n}-M_{i r r}\right)}+c \frac{\partial M_{a n}}{\partial H} .
$$

It is noted that in (5), the parameter $\widetilde{\alpha}\left(M_{i r r}, \sigma\right)$ represents an effective coupling coefficient that combines the interdomain coupling $\alpha$ and the magnetoelastic interactions,

$$
\widetilde{\alpha}\left(M_{i r r}, \sigma\right)=\alpha+\frac{3}{2 \mu_{0}} \frac{\partial^{2}(\sigma \varepsilon)}{\partial M_{i r r}^{2}} .
$$

\section{Constant-Field Magnetization}

We now consider the changes in magnetization that a magnetostrictive material undergoes when subjected to a uniaxial stress, or magnetomechanical effect $\partial M / \partial \sigma$. Although extensive experimental evidence suggests that the main mechanism governing the magnetomechanical effect is the unpinning of domain walls produced upon application of stress, at present no single model theory completely describes the observed behaviors. One description that has proved effective in the analysis of magnetostrictive transducers has been proposed by Jiles [16]. On the basis of a key model assumption that hysteresis originates primarily from domain wall pinning, the freeing of domain walls from their pinning sites is assumed to cause the magnetization to change in such a way as to approach the anhysteretic magnetization.

Experimental measurements demonstrate that both the magnitude and the direction of stressinduced magnetization changes are profoundly influenced by the magnetic history of the specimen $[16,20,23]$. It has been observed that the direction in which the magnetization changes with applied stress is independent of the sign of the stress for small stresses and when the magnetization is sufficiently distant from the anhysteretic. It is then inferred that the direction of change is dependent not on the stress itself, but on a quantity which is independent of the sign of the stress. In this context, it has been hypothesized in [16] that this quantity is the elastic energy per unit 
volume, $W=\sigma^{2} /(2 E)$, which is always independent of the sign of $\sigma$. The 'law of approach' to the anhysteretic state is then formulated as follows: the rate of change of magnetization with elastic energy is proportional to the displacement of the prevailing magnetization from the anhysteretic magnetization, or $\partial M / \partial W \propto M-M_{a n}$. The concept of the law of approach is here applied to the stress-induced magnetization of a magnetostrictive material.

As in the constant-stress magnetization case, the law of approach is modeled through irreversible and reversible components. It is noted that to a first approximation, the application of stress produces irreversible magnetization changes since $\Delta M$ arising from stress unloading is negligible. Thus, the law of approach is formulated in terms of the irreversible magnetization $M_{i r r}$,

$$
\frac{\partial M_{i r r}}{\partial W}=\frac{1}{\xi}\left(M_{a n}-M_{i r r}\right)
$$

where $\xi$ is a coefficient, having dimensions of energy per unit volume, that needs to be identified for magnetostrictive materials. Application of the chain rule $\partial M_{i r r} / \partial W=\left(\partial M_{i r r} / \partial \sigma\right)(\partial \sigma / \partial W)$ in (6), in combination with the relation $\partial W / \partial \sigma=\sigma / E$, yields

$$
\frac{\partial M_{i r r}}{\partial \sigma}=\frac{\sigma}{E \xi}\left(M_{a n}-M_{i r r}\right) .
$$

A similar argument to that used in the field-induced case yields the reversible component,

$$
\frac{\partial M_{r e v}}{\partial \sigma}=c\left(\frac{\partial M_{a n}}{\partial \sigma}-\frac{\partial M_{i r r}}{\partial \sigma}\right) \text {. }
$$

It is noted that the reversibility coefficient $c$ is the same as that defined in (4) because the energy available for domain wall bulging should be independent of the mechanism that produces the bulging, which can be either field- or stress-induced.

Summing the irreversible and reversible contributions given by (7) and (8) gives

$$
\frac{\partial M}{\partial \sigma}=(1-c) \frac{\sigma}{E \xi}\left(M_{a n}-M_{i r r}\right)+c \frac{\partial M_{a n}}{\partial \sigma},
$$

which is used to quantify the magnetomechanical effect in a magnetostrictive material. Equation (9) characterizes the law of approach from prevailing magnetization values above or below the anhysteretic curve. As depicted in Figure 2(a), changes in magnetization will be negative or positive depending on the position of the starting point $M_{0}$ relative to the anhysteretic curve. If the starting point is $A, \Delta M$ is negative and the relation between magnetization and stress is as given in inset (b), top. Conversely, starting point $B$ leads to positive values of $\Delta M$, and the magnetization traverses a curve like the one given in inset (b), bottom. It is noted that in both cases the field remains fixed at a value $H_{0}$. Figure 3 illustrates the shearing of the magnetization with increasing compressive stress. Consistently with experimental measurements on ferromagnetic materials, the law of approach described by relation (9) is such that when a magnetostrictive material is subjected to uniaxial compressive or tensile stress, the magnetization tries to approach the anhysteretic curve while the location of this curve changes as the stress is applied. Further details on this subject are given in [6].

It is noted that on application of stress the magnetization approaches a state of global energy equilibrium. This implies that the anhysteretic magnetization $M_{a n}$ must in this case be quantified by iteration of the Langevin function (2) until a solution which satisfies the equation identically is found. Further details regarding the differences between local and global solutions for equation (2) can be found in $[6]$. 


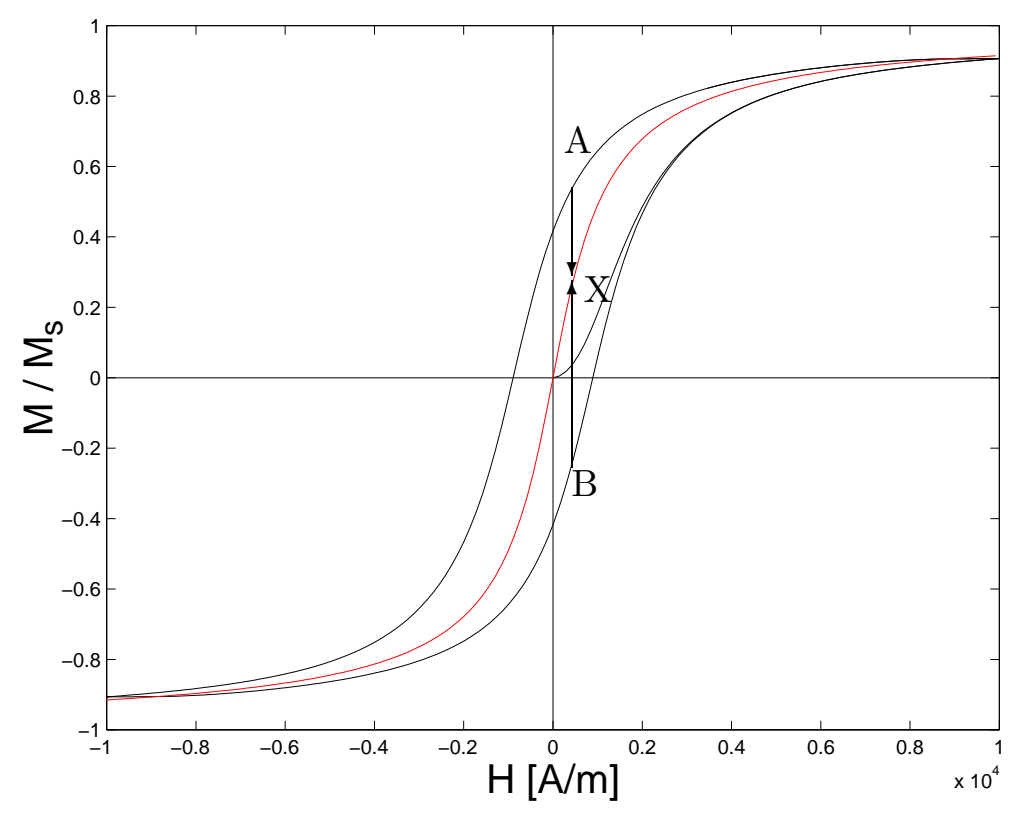

(a)
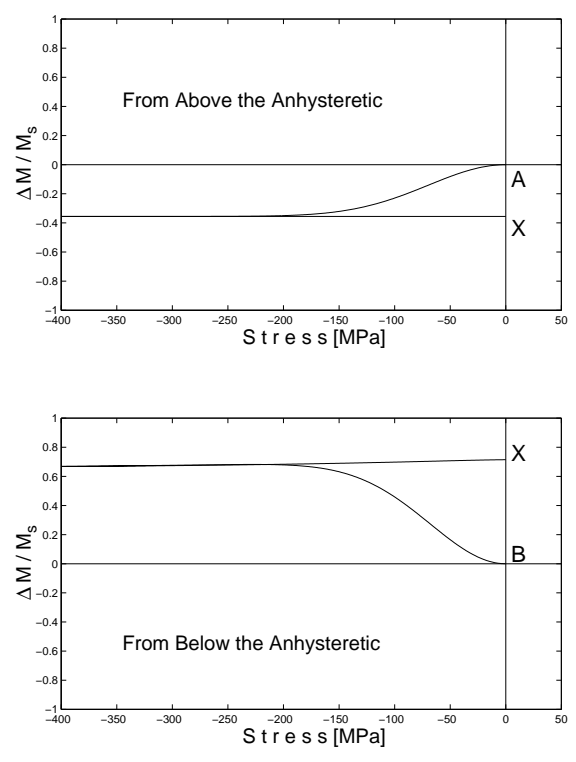

(b)

Figure 2: Representation of the approach to the anhysteretic under stress, after [20] (a) Arrows indicate law of approach from starting positions $M_{0}=A$ and $M_{0}=B$ above and below the anhysteretic curve, for fixed $H=H_{0}$. In either case, $M$ moves towards point $\mathrm{X}$ on the anhysteretic curve. (b) Trajectory of magnetization change $\Delta M / M_{s}$ upon application and further removal of a compressive stress, starting at point $\mathrm{A}(\mathrm{A} \rightarrow \mathrm{X})$, and at point $\mathrm{B}(\mathrm{B} \rightarrow \mathrm{X})$.
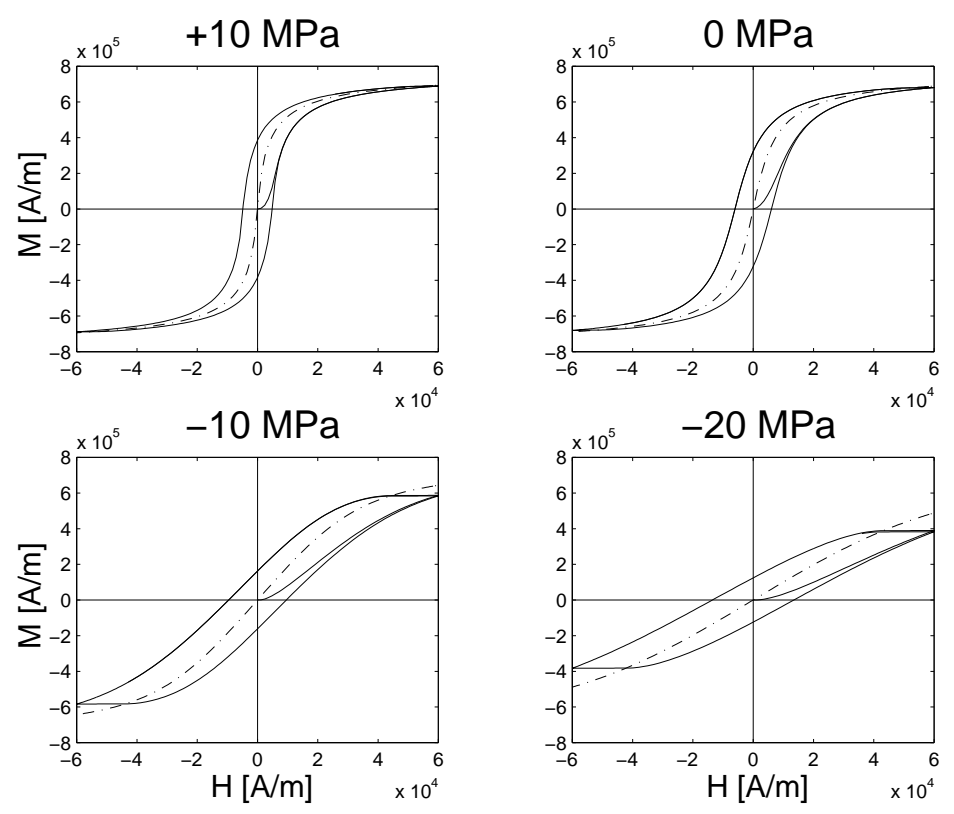

Figure 3: Model results of the effect of stress on the total magnetization $(-)$, and on the global anhysteretic magnetization $(-\cdot-)$, for $+10,0,-10$, and $-20 \mathrm{MPa}$. A positive magnetostriction coefficient is assumed. 


\section{MAGNETOSTRICTION}

In order to quantify the contribution of stress to the magnetization given by relation (9), it is necessary to characterize the strain and stress states in a magnetostrictive rod. To this end, it is necessary to consider first the deformations that a magnetostrictive material undergoes when its domain configuration changes. Several models exist for quantifying these deformations, including phenomenological formulations [16], a quadratic law for domain magnetization rotation [4], energy or thermodynamic formulations [23-25], elastomagnetic models [26-30], micromagnetic theories [14] and magnetization rotation analysis [3]. At low to moderate operating levels, or when material stresses are invariant, these deformations dominate over other material elastic dynamics. In such cases, it is theoretically possible to quantify the bulk magnetostriction upon knowledge of the domain configuration and the magnetostriction along easy crystallographic axes. In the case of Terfenol-D, nominal values for the latter are $\lambda_{111}=1600 \times 10^{-6}$ and $\lambda_{100}=90 \times 10^{-6}$, and $\lambda_{s} \approx 1000 \times 10^{-6}$. In practical terms, however, the domain configuration cannot be known a priori.

To motivate the approach followed here, we consider the particular case when the magnetic field is applied perpendicular to the axis in which the magnetic moments have been aligned by application of sufficiently large compression in the case of a polycrystalline material such as Terfenol-D, or perpendicular to the easy crystallographic axis in a single crystal with uniaxial anisotropy. In either case domain rotation is the prevailing magnetization mechanism, and the magnetostriction along the field direction is given by [4]

$$
\lambda(M)=\frac{3}{2} \lambda_{s}\left(\frac{M}{M_{s}}\right)^{2},
$$

which predicts a quadratic relationship between $\lambda$ and $M$. Relation (10) is single valued, while extensive experimental evidence suggests that the $\lambda-M$ relationship exhibits some degree of hysteresis. For transducer modeling purposes, it is feasible to utilize a single valued $\lambda$ - $M$ functional to model the overall shape, and to let $M$ provide the path dependences through the hysteretic mechanisms in $M-H$. This approach has proven effective in previous investigations [7].

It is noted that (10) is not sufficiently general when domain wall motion is significant, such as when the operating stress acting on the Terfenol-D material is not extreme $\left(\sigma_{0}<-6.9\right.$ to $\left.-20.7 \mathrm{MPa}\right)$. In order to provide a more general magnetostriction model, we consider a series expansion symmetric about $M=0$,

$$
\lambda(M)=\sum_{i=0}^{\infty} \gamma_{i} M^{2 i},
$$

in which the coefficients $\gamma_{i}$ need to be identified from experimental data. It is noted that quadratic relation (10) is obtained by assuming $i=1$ with $\gamma_{0}=0$ and $\gamma_{1}=\left(3 \lambda_{s}\right) /\left(2 M_{s}^{2}\right)$. For implementation purposes, we consider in this study a quartic law in which the series is truncated after $i=2$,

$$
\lambda(M)=\gamma_{1} M^{2}+\gamma_{2} M^{4} .
$$

\section{MAGNETOELASTIC COUPLING}

The magnetostriction relation (11) represents the strain arising from the reorientation of magnetic moments toward the direction of applied bias field $H_{0}$. It was shown in [7] that $\lambda$ provides a 
generalization of the term $d_{33} H$ in linear models of the form $\varepsilon=s^{H} \sigma+d_{33} H$. Equation (11) ignores, however, the elastic properties of a magnetostrictive material as it vibrates, as represented in linear models by $s^{H} \sigma$. Here, a PDE system is employed to quantify the elastic response of a magnetostrictive rod and relevant components located in a transducer's load path. The input to this system consists of the magnetostriction $\lambda$ and the external force $F_{\text {ext }}$ acting on a sensor. The solution to the PDE is the longitudinal displacements $u(t, x)$ relative to the prestressed position.

To formulate a model for the structural dynamics of a magnetostrictive sensor, we consider in Figure 4 a magnetostrictive rod, prestress bolt, prestress washer, and mass load following the constructive details of Figure 1. It is noted that the arbitrary external force acting on the rod is denoted $F_{\text {ext }}(t)$. The prestress bolt provides a stress $\sigma_{0}<0$ by compressing the magnetostrictive rod against the washer, modeled by linear spring $k_{L}$ and dashpot $c_{L}$. The rod is assumed to have length $L$, cross sectional area $A$, and longitudinal coordinate $x$. The material density is $\rho$, the elastic modulus is $E$, the internal (Kelvin-Voigt) damping is $c_{D}$, and the external load is modeled by a point mass $m_{L}$. It is emphasized that the value of parameter $E$ lies between that of the elastic modulus at constant $H, E^{H}$, and at constant $B, E^{B}$. Since $E^{H}$ and $E^{B}$ depend upon the field intensity [5], so does $E$. However, for simplicity $E$ is treated as a nominal or operational material stiffness.

Assuming linear elasticity and small displacements, force balancing yields a wave equation for the rod vibrations,

$$
\rho \frac{\partial^{2} u}{\partial t^{2}}(t, x)=\frac{\partial \sigma}{\partial x}(t, x)
$$

Here, the stress at cross sections $x$ in the rod is given by [7]

$$
\sigma(t, x)=E \frac{\partial u}{\partial x}(t, x)+c_{D} \frac{\partial^{2} u}{\partial x \partial t}(t, x)-E \lambda(t, x)+\sigma_{0},
$$

where $\lambda$ is given by (11) and $\sigma_{0}$ is the applied prestress. When integrated over a cross section, relation (12) yields the total inplane resultant $N(N>0$ in tension, $N<0$ in compression),

$$
N(t, x)=E A \frac{\partial u}{\partial x}(t, x)+c_{D} A \frac{\partial^{2} u}{\partial x \partial t}(t, x)-E A \lambda(t, x)+A \sigma_{0}
$$

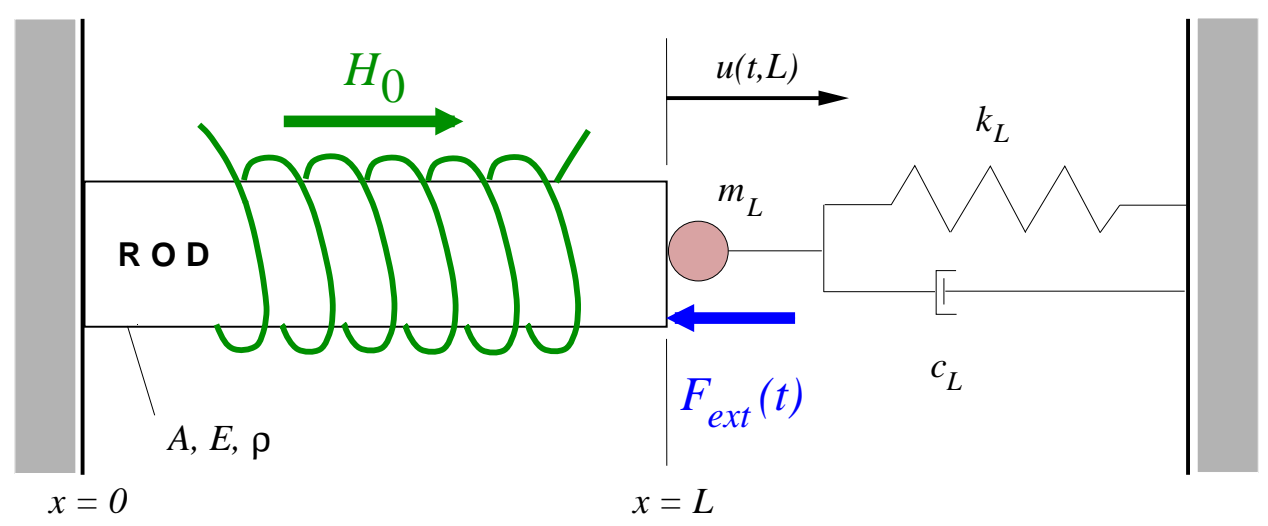

Figure 4: Schematic illustration of Villari-effect magnetostrictive sensor subjected to external force $F_{\text {ext }}(t)$. 
To obtain appropriate boundary conditions, it is first noted that at the fixed end of the rod $u(t, x)=$ 0 . At the end $x=L$, force balancing over an infinitesimal cross section of the rod yields

$$
N(t, L)=-m_{L} \frac{\partial^{2} u}{\partial t^{2}}(t, L)-c_{L} \frac{\partial u}{\partial t}(t, L)-k_{L} u(t, L)-F_{\text {ext }}(t) .
$$

The negative sign implies that $F_{\text {ext }}>0$ produces a compressive force in the magnetostrictive rod.

For implementation purposes, the model is formulated in weak or variational form by multiplying the strong form by test functions $\phi$ followed by integration throughout the length of the rod. This reduces the smoothness requirements on the finite element basis since displacements and test functions need to be differentiated only once compared to the second derivatives present in the strong form. The space of test functions is $V=H_{L}^{1}(0, L) \equiv\left\{\phi \in H^{1}(0, L) \mid \phi(0)=0\right\}$, so that for all $\phi(x) \in V$,

$$
\begin{aligned}
\int_{0}^{L} \rho A \frac{\partial^{2} u}{\partial t^{2}}(t, x) \phi(x) d x= & -\int_{0}^{L}\left[c_{D} A \frac{\partial^{2} u}{\partial x \partial t}(t, x)+E A \frac{\partial u}{\partial x}(t, x)-E A \lambda(t, x)\right] \frac{\partial \phi}{\partial x}(x) d x \\
& -\left[m_{L} \frac{\partial^{2} u}{\partial t^{2}}(t, L)+c_{L} \frac{\partial u}{\partial t}(t, L)+k_{L} u(t, L)+F_{\text {ext }}(t)\right] \phi(L) .
\end{aligned}
$$

The solution $u(t, x)$ to this equation defines the longitudinal displacements about the prestressed position and completely defines the elastic state through the strain, given by $\varepsilon(t, x)=\partial u / \partial x(t, x)$, and the stress $\sigma(t, x)$, given by (12). Iterative computation of stress and magnetization based on relations (9), (12) and (14) yields a fully coupled quantification of the magnetoelastic state of a magnetic field or force sensor.

\section{MODEL SUMMARY}

The magnetoelastic model presented here characterizes the behavior of a magnetostrictive sensor in response to two excitations: (i) a bias magnetic field $H_{0}$ applied at a known rate $d H / d t$ and (ii) a stress $\sigma$ originated from both the externally applied force and the strain produced by the material as it is magnetized. It is emphasized that in this formulation the magnetic and elastic regimes, represented by $M$ and $\sigma$ respectively, are coupled in accordance with the bidirectional energy transduction process exhibited by magnetostrictive materials. The model addresses both the actuation and sensing regimes by means of a unified mechanism. In actuator mode, externally applied magnetic fields produce magnetization changes which lead to strains and forces produced by a transducer as it drives external loads. In sensor mode, externally applied forces produce

magnetization changes which can be detected through the emf created in a sensing coil wrapped around a magnetostrictive rod. The model quantifies the relationship between input and output in either case.

In the presence of a magnetic field $H$ and a stress distribution $\sigma$, the magnetization of a magnetostrictive rod is dictated by the superposition of the field- and stress-dependent components given 
by (5) and (9),

$$
\begin{aligned}
\frac{d M}{d t}(t, x)= & \left\{(1-c) \frac{M_{a n}(t, x)-M_{i r r}(t, x)}{\delta k-\widetilde{\alpha}\left(M_{i r r}, \sigma\right)\left(M_{a n}(t, x)-M_{i r r}(t, x)\right)}+c \frac{\partial M_{a n}}{\partial H}(t, x)\right\} \frac{d H}{d t}(t, x) \\
& +\left\{(1-c) \frac{\sigma(t, x)}{E \xi}\left(M_{a n}(t, x)-M_{i r r}(t, x)\right)+c \frac{\partial M_{a n}}{\partial \sigma}(t, x)\right\} \frac{d \sigma}{d t}(t, x) .
\end{aligned}
$$

To characterize $d H / d t$, it is necessary to quantify first the field $H(t, x)$ generated by a solenoid when a current $I(t)$ circulates through it. It is often assumed that $H(t)=$ (No. turns /length) $I(t)$. However, this model is only valid in the idealized case of a lossless, infinitely long solenoid in a lossless magnetic circuit. A more accurate modeling approach consists of identifying $H-I$ by solving numerically Ampère's law or the Biot Savart law using, for example, finite element methods. For purposes of implementing the proposed model, the $H-I$ relationship is determined experimentally. The corresponding solenoid model is then written in the form,

$$
H(t, x)=N_{s} \Psi I(t)
$$

where $N_{s}$ is the number of turns in the solenoid and parameter $\Psi$, which needs to be measured from experimental data, is employed to account for solenoid end effects, demagnetizing factors, ohmic losses and flux leakage.

Upon substitution of (16) into (15), the final form for the time rate of change of magnetization is determined,

$$
\begin{aligned}
\frac{d M}{d t}(t, x)= & \left\{(1-c) \frac{M_{a n}(t, x)-M_{i r r}(t, x)}{\delta k-\widetilde{\alpha}\left(M_{i r r}, \sigma\right)\left(M_{a n}(t, x)-M_{i r r}(t, x)\right)}+c \frac{\partial M_{a n}}{\partial H}(t, x)\right\} N_{s} \Psi \frac{d I}{d t}(t) \\
& +\left\{(1-c) \frac{\sigma(t, x)}{E \xi}\left(M_{a n}(t, x)-M_{i r r}(t, x)\right)+c \frac{\partial M_{a n}}{\partial \sigma}(t, x)\right\} \frac{d \sigma}{d t}(t, x),
\end{aligned}
$$

which yields $M(t, x)$ upon integration. It should be noted that in the case of constant stress $(d \sigma / d t=0)$ or constant field $(d I / d t=0)$, the expression reduces to the individual components characterized by expressions (5) and (9).

After the magnetization $M(t, x)$ arising from the application of $H(t, x)$ and $\sigma(t, x)$ has been identified, active strains are computed from relation (11),

$$
\lambda[M(t, x)]=\gamma_{1} M^{2}(t, x)+\gamma_{2} M^{4}(t, x),
$$

where it is noted that since $\lambda$ depends on the applied magnetic field, it is not homogeneous throughout the rod. Hence, the magnetostriction varies along $x$.

The longitudinal rod displacements $u(t, x)$ are computed from (14)

$$
\begin{aligned}
\int_{0}^{L} \rho A \frac{\partial^{2} u}{\partial t^{2}}(t, x) \phi(x) d x= & -\int_{0}^{L}\left[E A \frac{\partial u}{\partial x}(t, x)+c_{D} A \frac{\partial^{2} u}{\partial x \partial t}(t, x)-E A \lambda(t, x)\right] \frac{\partial \phi}{\partial x}(x) d x \\
& -\left[F_{e x t}(t)+k_{L} u(t, L)+c_{L} \frac{\partial u}{\partial t}(t, L)+m_{L} \frac{\partial^{2} u}{\partial t^{2}}(t, L)\right] \phi(L) .
\end{aligned}
$$


To approximate the solution to this equation, a Galerkin discretization in $x$ is used to obtain a temporal system which is then solved with finite difference approximations. Details regarding this solution method are provided in [6]. Once the displacements have been characterized, the strains are computed directly through the relation

$$
\varepsilon(t, x)=\frac{\partial u}{\partial x}(t, x)
$$

and the corresponding stresses acting on the rod are computed directly from the strain using (12),

$$
\sigma(t, x)=E \frac{\partial u}{\partial x}(t, x)+c_{D} \frac{\partial^{2} u}{\partial x \partial t}(t, x)-E \lambda(t, x)+\sigma_{0} .
$$

\section{EXAMPLE}

Relation (18) is now employed to characterize the magnetization changes produced by a Terfenol-D sensor with configuration as illustrated in Figure 1 in response to an externally applied force. The force was generated with a PZT-5A piezoelectric stack and its magnitude was measured with a PCB 086C03 load cell arranged as indicated in Figure 5. The complete device was rigidly clamped to the wall at each end.

The measured output from the sensor during operation included the sensing voltage $V(t)$ and impressed force $F_{\text {ext }}(t)$. The prestress level in the magnetostrictive rod was $\sigma_{0}=-3.45 \mathrm{MPa}$. A bias field of magnitude $H_{0}=75.8 \mathrm{kA} / \mathrm{m}$ was applied with an Alnico V permanent magnet which was slit longitudinally to reduce eddy current losses. Steel end caps and a Belleville compression washer completed the magnetic circuit.

The magnetostrictive material was a $50 \mathrm{~mm}$ long, $6.35 \mathrm{~mm}$ diameter monolithic $\mathrm{Tb}_{0.3} \mathrm{Dy}_{0.7} \mathrm{Fe}_{1.92}$ rod manufactured by the Free Stand Zone Melt process. The sensing signal was provided by a 1100turn solenoid wound with AWG26 magnet wire. The magnetic induction $B_{p u}(t)$ was calculated by integration of the sensing signal $V(t)$. Following the Faraday-Lenz law of magnetic induction, $B_{p u}(t)=-1 /\left(N_{p u} A_{p u}\right) \int_{0}^{t} V(\tau) d \tau$. Here, $A_{p u}$ is the mean cross sectional area and $N_{p u}$ is the

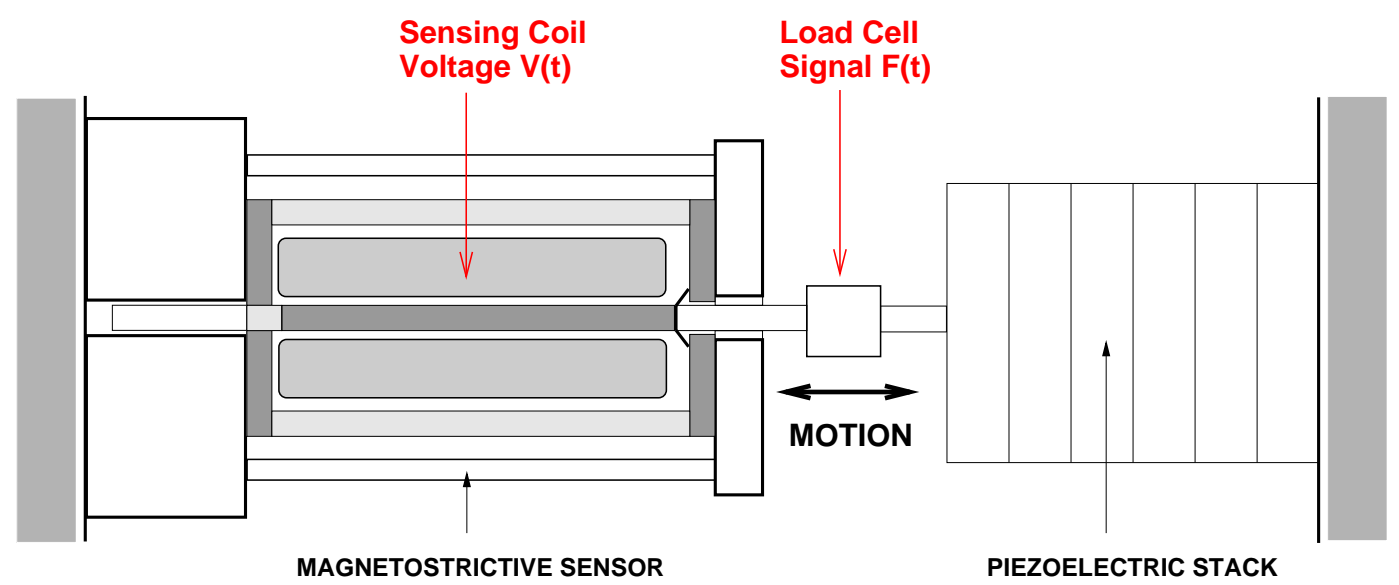

Figure 5: Schematic representation of the assembly used to drive the magnetostrictive sensor. Measured input was driving force $F(t)$, while measured output was sensing voltage $V(t)$. 
number of turns of the sensing solenoid. Figures 6(a-c) show respectively the $50 \mathrm{~Hz}$ applied force, sensing voltage and magnetic induction data obtained in the case of $550 \mathrm{~V}$ volts applied to the PZT stack.

For simulation purposes, the material was magnetized by applying a quasistatic $(1 \mathrm{~Hz})$ sinusoidal current until a prescribed bias field value $H_{0}$ was reached. The field value remained unchanged thereafter to ensure a constant magnetization bias during operation. The magnetic induction was calculated from the model magnetization $M$ and the applied field $H$ via the magnetic constitutive relation $B=\mu_{0}(M+H)$, where $\mu_{0}$ is the permeability of free space. It is noted that while the derivative $d B / d t$ provides a characterization of the sensing voltage through $V=-N_{p u} A_{p u} d B_{p u} / d t$, derivatives of quantities involving experimental data typically exhibit significant noise, thus precluding a proper comparison of measured and calculated sensing voltages. For this reason, a comparison is established between measured and calculated induction, $B_{p u}$ and $B$, respectively.

Basic properties of the model are illustrated in Figure 6(d). The model provides a very accurate representation of the magnetic induction in the magnetostrictive rod in both the shape and amplitude of the measured response. The minor phase lag of the model with respect to the data may be explained by eddy current losses in the rod arising due to the $50 \mathrm{~Hz}$ frequency of operation. Use of a laminated sample will surely help to decrease the observed lag.

\section{CONCLUDING REMARKS}

A magnetomechanical model for quantifying the behavior of magnetostrictive materials as used in Villari-effect sensors has been presented. The model addresses the bidirectional energy transduction between the magnetic and elastic regimes by means of a coupling mechanism posed in terms of a PDE system. This PDE system treats the case of a magnetostrictive material driving external loads (actuator mode) or being driven by external loads (sensor mode). Although some model components are ultimately based on phenomenological observation, crucial aspects of the model are constructed from thermodynamic principles. For this reason, it is expected that a near-constant set of parameters will provide accurate characterization of sensor performance over a wide range of regimes, including highly nonlinear regimes where prior models provide inaccurate results.

The example demonstrated the use of the model to quantify the magnetic induction changes exhibited by a magnetically biased and mechanically preloaded Terfenol-D rod subjected to external forces. This example provides a template for applications based on magnetostrictive materials in which the induction changes created in a magnetostrictive rod are used to generate voltages in a surrounding sensing coil. It was shown that the model accurately characterizes the relationship between input force and output magnetic induction under typical conditions.

\section{ACKNOWLEDGMENT}

Financial support for A.B.F. and M.J.D. was provided by the NSF Young Investigator Award \#CMS9457288 of the Division of Civil and Mechanical Systems and by The Ohio State University. The work of R.C.S. was supported in part by the Air Force Office of Scientific Research under the grant AFOSR F49620-01-1-0107. 


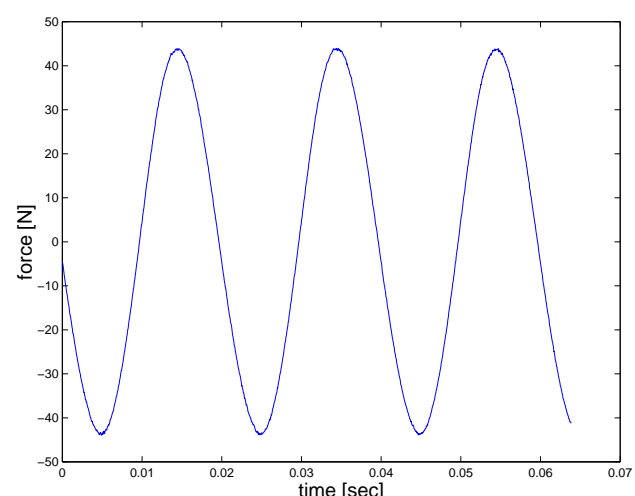

(a)

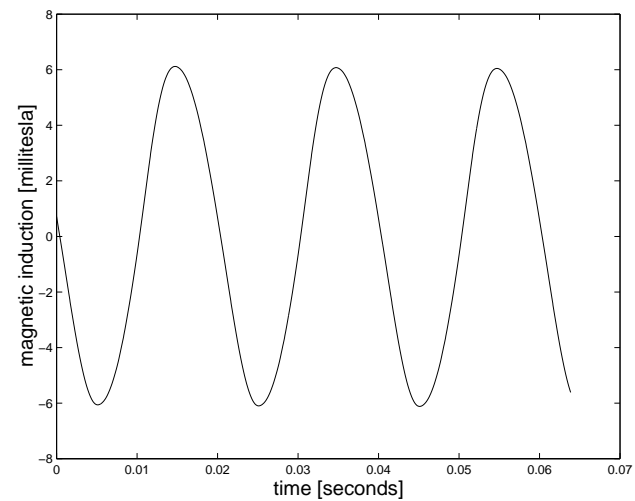

(c)

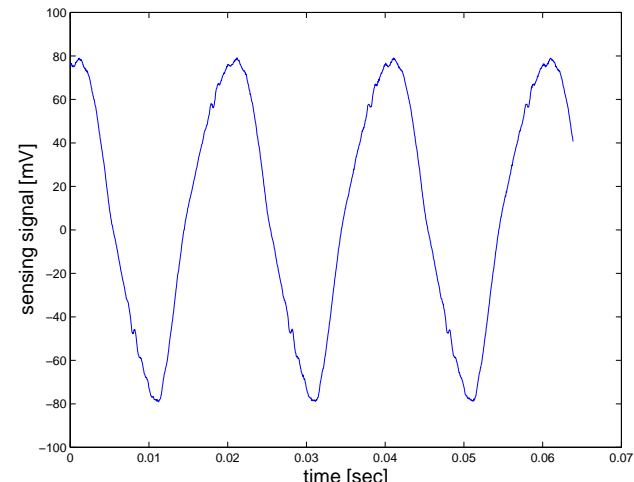

(b)

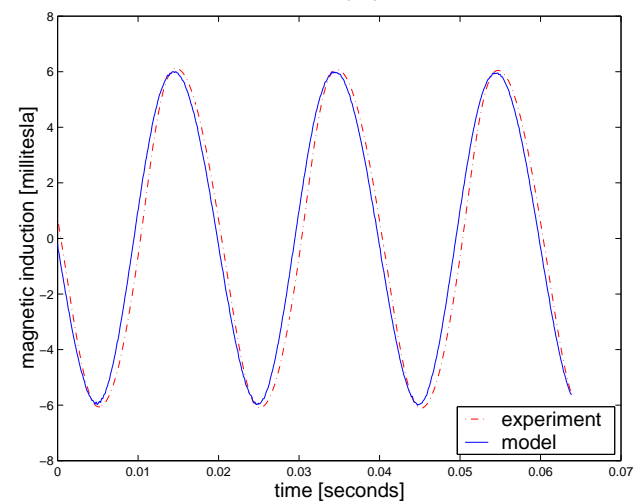

(d)

Figure 6: Experimental data: (a) force applied to sensor, (b) sensing voltage from sensor and (c) magnetic induction computed from (b). Model performance: (d) comparison of magnetic induction from model with data shown in (c). The parameters used for model simulation are: $a=7000 \mathrm{~A} / \mathrm{m}$, $k=7000 \mathrm{~A} / \mathrm{m}, c=0.2, \alpha=0.065, E=40 \mathrm{GPa}, \rho=9250 \mathrm{Kg} / \mathrm{m}^{3}, \gamma_{1}=2.95 \times 10^{-15} \mathrm{~m}^{2} / \mathrm{A}^{2}$, $\gamma_{2}=-6 \times 10^{-28} \mathrm{~m}^{4} / \mathrm{A}^{4}, \xi=8 \times 10^{3} \mathrm{~Pa}, c_{D}=1 \times 10^{6} \mathrm{Ns} / \mathrm{m}, c_{L}=1 \times 10^{3} \mathrm{Ns} / \mathrm{m}, k_{L}=2.66 \times 10^{6} \mathrm{~N} / \mathrm{m}$, $m_{L}=0.1 \mathrm{Kg}, \sigma_{0}=-3.45 \mathrm{MPa}, L=50 \mathrm{~mm}, D=6.35 \mathrm{~mm}$.

\section{REFERENCES}

[1] Atherton, D.L. and V. Ton. 1990. "Effect of order of stress and field application on changes in anhysteretic magnetization," IEEE Transactions on Magnetics, 26(3):1157-1159.

[2] Chung, R., R. Weber and D. Jiles. 1991. "A Terfenol-D based magnetostrictive diode laser magnetometer," IEEE Trans. Magn., 27(6):5358-5243.

[3] Clark, A.E., H.T. Savage and M.L. Spano. 1984. "Effect of stress on the magnetostriction and magnetization of single crystal $\mathrm{Tb}_{0.27} \mathrm{Dy}_{0.73} \mathrm{Fe}_{2}, "$ IEEE Trans. Magn., MAG-20(5).

[4] Cullity, B.D. 1972. "Introduction to magnetic materials," Addison-Wesley, Reading, Massachusetts.

[5] Dapino, M.J., A.B. Flatau and F.T. Calkins. 1997. "Statistical analysis of Terfenol-D material properties," Proceedings of SPIE, Smart Structures and Materials 1997, 3041:256-267, San Diego, CA. 
[6] Dapino, M.J., R.C. Smith, L.E. Faidley and A.B. Flatau. 2000. "A coupled structuralmagnetic strain model for magnetostrictive transducers," J. Intell. Mater. Sys. Struct., 11:135-152.

[7] Dapino, M.J., R.C. Smith and A.B. Flatau. 2000. "Structural-magnetic strain model for magnetostrictive transducers," IEEE Trans. Magn., 36(3): 545-556.

[8] Doherty, J., S. Arigapudi and R. Weber. 1994. "Spectral estimation for a magnetostrictive magnetic field sensor," IEEE Trans. Magn., 30(3):1274-1290.

[9] Engdahl, G. (Ed). 2000. "Handbook of giant magnetostrictive materials," Academic Press, San Diego, CA.

[10] Fenn, R. and M. Gerver. 1994. "Passive damping and velocity sensing using magnetostrictive transduction," Proceedings of SPIE, Smart Structures and Materials 1994, 2190:216-227.

[11] Fleming, W. 1989. "Magnetostrictive torque sensor performance - nonlinear analysis," IEEE Trans. Vehic. Tech., 38(3):159-167.

[12] Garshelis, I.J. and C.R. Conto. 1996. "A magnetoelastic torque transducer utilizing a ring divided into two oppositely polarized circumferential regions," J. Appl. Phys. 79(8): 47564758 .

[13] Hunt, F.V. 1982. "Electroacoustics: the analysis of transduction, and its historical background," American Institute of Physics for the Acoustical Society of America.

[14] James, R.D. and D. Kinderlehrer. 1993. "Theory of magnetostriction with applications to $\mathrm{Tb}_{\mathrm{x}} \mathrm{Dy}_{1-\mathrm{x}} \mathrm{Fe}_{2}, "$ Philosophical Magazine B, 68(2):237-274.

[15] Jiles, D.C. and D.L. Atherton. 1986. "Theory of ferromagnetic hysteresis," J. Magn. Magn. Mater., 61:48-60.

[16] Jiles, D.C. 1995. "Theory of the magnetomechanical effect," J. Phys. D: Appl. Phys., 28:15371546.

[17] Jiles, D.C. 1998. "Introduction to Magnetism and Magnetic Materials," Chapman \& Hall, London.

[18] Jones, L. and E. Garcia. 1994. "Application of the self-sensing principle to a magnetostrictive structural element for vibration suppression," ASME Proc. Int. Mech. Eng. Congr. Expos., 45, pp. 155-165, Chicago.

[19] Kleinke, D.K. and H.M Uras. 1996. "Modeling of magnetostrictive sensors," Rev. Sci. Instrum., 67(1):294-301.

[20] Pitman, K.C. 1990. "The influence of stress on ferromagnetic hysteresis," IEEE Trans. Magn., 26(5):1978-1980.

[21] Pratt, J. and A.B. Flatau. 1995. "Development and analysis of a self-sensing magnetostrictive actuator design," Journal of Intelligent Material Systems and Structures, 6(5):639-648.

[22] Ristic, L. 1994. "Sensor technology and devices," Artech House, Inc., Norwood, MA. 
[23] Sablik, M.J. 1997. "A model for asymmetry in magnetic property behavior under tensile and compressive stress in steel," IEEE Transactions on Magnetics, 33(5):3958-3960.

[24] Sablik, M.J. and D.C. Jiles. 1999. "Modeling the effects of torsional stress and hysteretic magnetization," IEEE Transactions on Magnetics, 35(1):498-504.

[25] Seekercher, J. and B. Hoffmann. 1990. "New magnetoelastic force sensor using amorphous alloys," Sensors Actuators, A21-23:401-405.

[26] Trémolet de Lacheisserie, E. 1993. "Magnetostriction theory and applications of magnetoelasticity," CRC Press, Inc., Boca Raton, FL. 